International Journal of Pure and Applied Mathematics

Volume 102 No. 2 2015, 169-186

ISSN: 1311-8080 (printed version); ISSN: 1314-3395 (on-line version)

url: http://www.ijpam.eu

doi: http://dx.doi.org/10.12732/ijpam.v102i2.1

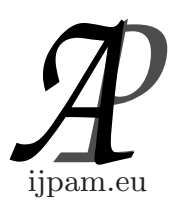

\title{
EXISTENCE OF GLOBAL SOLUTIONS FOR SYSTEMS OF REACTION-DIFFUSION WITH COMPACT RESULT
}

\author{
Abdelkader Moumeni ${ }^{1}$, Nabila Barrouk ${ }^{2} \S$ \\ ${ }^{1,2}$ Laboratoire de Mathématiques \\ Dynamique et Modélisation \\ Université Badji Mokhtar-Annaba \\ B.P. 12 Annaba 23000, ALGÉRIE \\ ${ }^{2}$ Department of Mathematics and Informatics \\ Faculty of Sciences \\ University of Souk Ahras \\ 41000, Souk Ahras, ALGERIA
}

\begin{abstract}
The aim of this paper is to study the global existence in time of solutions for some class of reaction-diffusion systems. Our techniques of proof is based on compact semigroup methods and some $L^{1}$ estimates. Our goal is to show, under suitable assumptions, that the proposed model have a global solution for a large class of the functions $f$ and $g$.
\end{abstract}

AMS Subject Classification: 35K57, 35K40, 35K55

Key Words: global solution, semi-groups, local solution, reaction-diffusion systems

\section{Introduction}

Recently, a class of systems of partial differential equations of the parabolic type, called system of reaction-diffusion, it received considerable interest by the

Received: December 18, 2014

(C) 2015 Academic Publications, Ltd.

$\S$ Correspondence author url: www.acadpubl.eu 
researchers, motivated by both the enrichment structure of the solution as well as it governs several chemical, ecological, biological, metallurgical phenomena and even in marketing.

These systems spell in their simplest shape as follows:

$$
\left.\frac{\partial w}{\partial t}-D \Delta w=F(w) ; \text { in }\right] 0,+\infty[\times \Omega
$$

where $\Omega$ is a bounded domain in $\mathbb{R}^{n}$ with boundary $\left.\partial \Omega, w:\right] 0,+\infty\left[\times \Omega \rightarrow \mathbb{R}^{2}\right.$, i.e. $w(t, x)=(u(t, x), v(t, x))$,

$$
F: \mathbb{R}^{2} \rightarrow \mathbb{R}^{2}, \quad F(w(t, x))=(f(w(t, x)), g(w(t, x)))
$$

is the term of the reaction (generally nonlinear). The terms of reaction are the result of any interaction between the constituents of the unknown $w$.

The objective of this work has contributed to the study of the global existence in times of the solution of (SRD) with Neumann boundary condition:

$$
\left.\frac{\partial w}{\partial \eta}=0 \quad \text { on }\right] 0,+\infty[\times \partial \Omega,
$$

and the initial data

$$
w(0, x)=w_{0}(x) \geq 0, \quad \text { in } \Omega .
$$

Most studies which are made about the system of reaction-diffusion is essentially based on some particular cases of (SRD), where the mathematical model:

$$
\begin{gathered}
\frac{\partial u}{\partial t}-d_{1} \Delta u=f(u, v) \\
\frac{\partial v}{\partial t}-d_{2} \Delta v=g(u, v) \\
\frac{\partial u}{\partial \eta}=\frac{\partial v}{\partial \eta}=0 \\
u(0, .)=u_{0}, v(0, .)=v_{0}
\end{gathered}
$$

where, $d_{1}$ and $d_{2}$ are two positive constants, is the most approached by the researchers.

When $f$ and $g$ are "enough regular" and $u_{0}, v_{0}$ are bounded, the local existence in times of the solution $(u, v)$ is classical. Furthermore, it is not negative if $u_{0}$ and $v_{0}$ too. 
The main question we want to address is the existence of global solutions for system (1.1)-(1.4). In fact, the subject of the global existence of reactiondiffusion systems has received a lot of attention in the last decades and several outstanding results have been proved by some of the major experts in the field. see [2], [3], [12].

This question has been investigated by many authors by considering special forms of the nonlinear terms $f$ and $g$. Note that, Alikakos [1], treated this system with the same boundary conditions (1.3) and initial condition (1.4), where $f(u, v)=-g(u, v)=-u v^{\sigma}$, and gave a positive answer when $1<\sigma<$ $\frac{n+2}{n}$ with method is based on some Sobolev embedding theorems.

In [15], Masuda obtained a global existence result for a large class of the parameter $\sigma$. In fact, by using some $L^{p}$ estimates, he showed that the solution of the problem (1.1)-(1.4) exists globally in time if $\sigma>1$.

Following Masuda's approach, Haraux and Youkana [5] established a global existence result of a system (1.1)-(1.4) for a large class of the function $f$ and $g$. More precisely, they showed that for

$$
f(u, v)=g(u, v)=-u \Psi(v)
$$

the problem (1.1)-(1.4) admits a global solution provided that the following condition holds:

$$
\lim _{v \rightarrow+\infty} \frac{[\log (1+\Psi(v))]}{v}=0 .
$$

The same result in [15] was obtained by Hollis, Martin and Pierre [8] by exploiting the duality of arguments in $L^{p}$ techniques, allowing to derive the uniform bounds of the solution.

In the general case, that is to say for

$$
f(u, v)=-g(u, v)
$$

the positivity of the function $g(u, v)$ together with the maximum principle of the heat operator give the following uniform estimate of the solution in $L^{\infty}(\Omega)$

$$
\|u(t)\|_{\infty} \leq\left\|u_{0}\right\|_{\infty}, \quad \forall t \in\left[0, T_{\max }[\right.
$$

where $T_{\max }$ is the maximal time of existence. See Pazy [17] for more details.

Based on the Lyapunov functional method and for $f$ and $g$ satisfying (1.5), Kouachi [11] proves that the solution of the problem (1.1)-(1.4) exists globally in time if

$$
\lim _{v \rightarrow+\infty} \frac{[\log (1+f(u, v))]}{v} \leq \frac{8 \alpha \beta}{n(\alpha-\beta)^{2}\left\|u_{0}\right\|_{\infty}}
$$


Recently, Moumeni and Salah Derradji [16] has established the existence of global solution using an approach that involves the Lyapunov's functional for the system (1.1)-(1.4) where the functions $f$ and $g$ are assumed to satisfy the condition

$$
\sup (|f(r, s)|,|g(r, s)|) \leq C(r+s+1)^{m}, \quad \forall r, s \geq 0
$$

where $C$ is a positive constant and $m \geq 1$.

If $d_{1} \neq d_{2}$, an important particular case is that when $f \leq 0$, which means that the first substance is absorbed by the reaction. In this case, the problem of the global existence of a system (1.1)-(1.2) is reduced to obtaining a uniform estimate for $v$, since by the maximal principle we have

$$
u(t, x) \leq\left\|u_{0}\right\|_{\infty} .
$$

The global existence when $d_{1}>d_{2}$ has been treated by Kanel and Kirane [9] for a bounded domain and by Martin and Pierre [14] for whole space $\mathbb{R}^{n}$.

Still for the case $d_{1} \neq d_{2}$, but without assuming $d_{1}>d_{2}$, the answer is again positive to the problem of the global existence of a system (1.1)-(1.2) under condition (1.6) and a polynomial growth assumption on $g$ :

$$
g(u, v) \leq C(u+v+1)^{\gamma}, \quad \text { for all } u, v \geq 0 \text { and some } \gamma \geq 1,
$$

see [8] for more details.

If the diffusion coefficients are the same, that is, if $d_{1}=d_{2}$, then system (1.1)-(1.2) has a global solution under the condition

$$
f(u, v)+g(u, v) \leq 0,
$$

which is known as the mass dissipative structure condition. Indeed if $d_{1}=d_{2}$, then the solution $(u, v)$ of (1.1)-(1.2) satisfies (by summing up the two equations in $(1.1)-(1.2))$

$$
\frac{\partial}{\partial t}(u+v)-d_{1}(u+v)=f+g \leq 0 .
$$

Then the maximal principle implies that:

$$
0 \leq u+v \leq\left\|u_{0}\right\|_{\infty}+\left\|v_{0}\right\|_{\infty} .
$$

Therefore, the global existence follows.

In the present work we consider the problem (1.1)-(1.4) by using a technique based on $L^{1}$-estimate we establish a global existence result of the solution. 


\subsection{Formulation of the result}

We consider the problem (1.1)-(1.4) where we suppose the following hypotheses

$$
\begin{gathered}
u_{0}, v_{0} \text { are nonnegative functions in } L^{1}(\Omega) \\
f(0, v) \geq 0, g(u, 0) \geq 0 ; \forall u, v \geq 0 . \\
\exists C \geq 0: f(u, v)+g(u, v) \leq C(u+v+1) ; \forall u, v \geq 0 . \\
\exists \hat{C} \geq 0: f(u, v) \leq \hat{C}(u+v+1) ; \forall u, v \geq 0 .
\end{gathered}
$$

The existence of global solutions for the system (1.1)-(1.4) is to equivalence to existence a $(u, v)$ true for the following theorem:

Theorem 1. Suppose that the hypotheses $\left(\boldsymbol{H}_{i}\right), i=\overline{1,4}$ are satisfied, so it exists $(u, v)$ solution of:

$$
\begin{cases}u, v \in C\left(\left[0,+\infty\left[, L^{1}(\Omega)\right)\right.\right. & \\ f(u, v), g(u, v) \in L^{1}(Q) \text { where } Q=(0, T) \times \Omega \text { for all } T>0, \\ u(t, x)=S_{1}(t) u_{0}+\int_{0}^{t} S_{1}(t-s) f(u(s), v(s)) d s, \quad \forall t \in[0, T[ \\ v(t, x)=S_{2}(t) v_{0}+\int_{0}^{t} S_{2}(t-s) g(u(s), v(s)) d s, \quad \forall t \in[0, T[\end{cases}
$$

where $S_{1}(t)$ and $S_{2}(t)$ are the Semigroups of contractions in $L^{1}(\Omega)$ generated by $d_{1} \Delta$ and $d_{2} \Delta$, with homogeneous Neumann boundary conditions.

To prove this theorem we will rely on studying a single system through which it is more convenient to derive the evidence.

\section{Main results}

Let $A$ m-dissipative operator of the dense domain in the Banach space $X$ and $S(t)$ a Semigroup engendered by $A, f$ a function locally Lipchitz, so $\forall u_{0} \in X$ it exists $T\left(u_{0}\right)=T_{\max }$ such that the problem

$$
\left\{\begin{array}{l}
u \in C([0, T], D(A)) \cap C^{1}([0, T], X), \\
\frac{d u}{d t}-A u=F(u(s)) \\
u(0)=u_{0} .
\end{array}\right.
$$


admits a unique solution $u$ verifying

$$
u(t)=S(t) u_{0}+\int_{0}^{t} S(t-s) F(u(s)) d s, \forall t \in\left[0, T_{\max }\right] .
$$

\section{Compactness of the solution}

In this section we will give a compactness result of operator $L$ defining the solution of the problem (2.1) in the case where the initial value equals zero $[u(0)=0]$ i.e.

$$
L(F)(t)=u(t)=\int_{0}^{t} S(t-s) F(u(s)) d s, \forall t \in[0, T]
$$

Theorem 2. If for all $t>0$, the operators $S(t)$ are compact, then $L$ are compact of $L^{1}([0, T], X)$ in $L^{1}([0, T], X)$.

Proof. Step 1: We show that $S(\lambda) L: F \rightarrow S(\lambda) L(F)$ is compact in $L^{1}([0, T], X)$ i.e. show that: the set $\left\{S(\lambda) L(F)(t) ;\|F\|_{1} \leq 1\right\}$ is relatively compact in $L^{1}([0, T], X), \forall t \in[0, T]$.

Since $S(t)$ is compact then, the application: $t \rightarrow S(t)$ is continuous of ] $0,+\infty[$ in $£(X)$ therefore:

$$
\forall \varepsilon>0, \forall \delta>0, \exists \eta>0 . \forall 0 \leq h \leq \eta, \forall t \geq \delta,\|S(t+h)-S(t)\|_{f(X)} \leq \varepsilon
$$

choose $\lambda=\delta$, we have for $0 \leq t \leq T-h$

$$
\begin{aligned}
S(\lambda) & u(t+h)-S(\lambda) u(t) \\
= & \int_{0}^{t+h} S(\lambda+t+h-s) F(u(s)) d s-\int_{0}^{t} S(\lambda+t-s) F(u(s)) d s \\
= & \int_{t}^{t+h} S(\lambda+t+h-s) F(u(s)) d s+\int_{0}^{t}(S(\lambda+t+h-s) \\
& -S(\lambda+t-s)) F(u(s)) d s
\end{aligned}
$$


where from

$$
\|S(\lambda) u(t+h)-S(\lambda) u(t)\|_{X} \leq \int_{t}^{t+h}\|F(u(s))\|_{X} d s+\varepsilon \int_{0}^{t}\|F(u(s))\|_{X} d s
$$

we definite $v(t)$ by

$$
v(t)= \begin{cases}u(t) & \text { for } 0 \leq t \leq T \\ 0 & \text { if no }\end{cases}
$$

therefore:

$$
\|S(\lambda) v(t+h)-S(\lambda) v(t)\|_{1} \leq(h+\varepsilon T)\|F(u(s))\|_{1}
$$

which implies that all $\left\{S(\lambda) v ;\|F\|_{1} \leq 1\right\}$ is equi-integrable, then it is conventional that all $\left\{S(\lambda) L(F)(t) ;\|F\|_{1} \leq 1\right\}$ is relatively compact in $L^{1}([0, T], X)$, this way $S(\lambda) L$ is compact.

Step 2: We show that $S(\lambda) L$ converge towards $L$ when $\lambda$ goes towards 0 , in $L^{1}([0, T], X)$.

We have:

$$
S(\lambda) u(t)-u(t)=\int_{0}^{t} S(\lambda+t-s) F(u(s)) d s-\int_{0}^{t} S(t-s) F(u(s)) d s .
$$

So for $t \geq \delta$ we have:

$\|S(\lambda) u(t)-u(t)\| \leq \int_{\delta}^{t}\|S(\lambda+s)-S(s)\|_{\mp(X)}\|F(u(s))\| d s+2 \int_{t-\delta}^{t}\|F(u(s))\| d s$

choose $0<\lambda<\eta$ then:

$$
\|S(\lambda) u(t)-u(t)\| \leq \varepsilon \int_{\delta}^{t}\|F(u(s))\| d s+2 \int_{t-\delta}^{t}\|F(u(s))\| d s
$$

and for $0 \leq t<\delta$ we have:

$$
\|S(\lambda) u(t)-u(t)\| \leq 2 \int_{0}^{t}\|F(u(s))\| d s
$$


as $F \in L^{1}(0, T, X)$ where from:

$$
\|S(\lambda) u(t)-u(t)\| \leq(\varepsilon T+2 \delta)\|F(u(s))\|_{1}
$$

so if $\lambda \rightarrow 0$ then $S(\lambda) u \rightarrow u$ into $L^{1}([0, T], X)$

where the operator $L$ is a uniform limit with compact linear operator between two Banach spaces, then $L$ is compact in $L^{1}([0, T], X)$.

Remark 1. The Semigroup $S(t)$ generated by the operator $\Delta$ is compact in $L^{1}(\Omega)$.

\section{Study of a particular system}

for all $n>0$, we define the functions $u_{n_{0}}$ and $v_{n_{0}}$ by:

$$
u_{n_{0}}=\min \left(u_{0}, n\right) \geq 0, \quad \text { and } \quad v_{n_{0}}=\min \left(v_{0}, n\right) \geq 0
$$

it is clear that $u_{n_{0}}$ and $v_{n_{0}}$ verify $\left(\mathrm{H}_{1}\right)$, i.e.

$$
\begin{array}{ll}
u_{n_{0}} \in L^{1}(\Omega), & u_{n_{0}} \geq 0 \\
v_{n_{0}} \in L^{1}(\Omega), & v_{n_{0}} \geq 0
\end{array}
$$

Let us consider the following system:

$$
\begin{cases}\frac{\partial u_{n}}{\partial t}-d_{1} \Delta u_{n}=f\left(u_{n}, v_{n}\right) & \text { in }[0, T[\times \Omega \\ \frac{\partial v_{n}}{\partial t}-d_{2} \Delta v_{n}=g\left(u_{n}, v_{n}\right) & \text { in }[0, T[\times \Omega \\ \frac{\partial u_{n}}{\partial \eta}=\frac{\partial v_{n}}{\partial \eta}=0 & \text { in }[0, T[\times \partial \Omega \\ u_{n}(0, x)=u_{n_{0}}(x), v_{n}(0, x)=v_{n_{0}}(x) & \text { in } \Omega,\end{cases}
$$

4.1. Existence of a local solution and its positivity of the solution of the system $\left(\mathbf{P}_{n}\right)$

We convert the system $\left(\mathrm{P}_{n}\right)$ to an abstract first order system in the Banach space $X=L^{1}(\Omega) \times L^{1}(\Omega)$ of the form

$$
\left\{\begin{array}{l}
\frac{\partial w_{n}}{\partial t}=A w_{n}+F\left(w_{n}\right), \quad t>0 \\
w_{n}(0)=w_{n_{0}}=\left(u_{n_{0}}, v_{n_{0}}\right) \in X
\end{array}\right.
$$

Here $w_{n}=\left(\begin{array}{c}u_{n} \\ v_{n}\end{array}\right)$; the operator $A$ is defined as

$$
A=\left(\begin{array}{cc}
d_{1} \Delta & 0 \\
0 & d_{2} \Delta
\end{array}\right)
$$


where $D(A):=\left\{w_{n}=\left(\begin{array}{c}u_{n} \\ v_{n}\end{array}\right) \in X:\left(\begin{array}{c}\Delta u_{n} \\ \Delta v_{n}\end{array}\right) \in X\right\}$

The function $F$ is defined as $F\left(w_{n}(t)\right)=\left(\begin{array}{l}f\left(u_{n}(t), v_{n}(t)\right) \\ g\left(u_{n}(t), v_{n}(t)\right)\end{array}\right)$.

so the system $\left(\mathrm{S}_{n}\right)$ can be returned to the shape of the system (2.1), thus, if $\left(u_{n}, v_{n}\right)$ is a solution of $\left(\mathrm{S}_{n}\right)$ so it verifies the integral equations:

$$
\left\{\begin{array}{l}
u_{n}(t, x)=S_{1}(t) u_{n_{0}}+\int_{0}^{t} S_{1}(t-s) f\left(u_{n}(s), v_{n}(s)\right) d s \\
v_{n}(t, x)=S_{2}(t) v_{n_{0}}+\int_{0}^{t} S_{2}(t-s) g\left(u_{n}(s), v_{n}(s)\right) d s
\end{array}\right.
$$

where $S_{1}(t)$ is the semigroup generated by the operator $d_{1} \Delta$, and $S_{2}(t)$ is the semigroup generated by the operator $d_{2} \Delta$.

Theorem 3. It exists $T_{M}>0$ and $\left(u_{n}, v_{n}\right)$ a local solution of $\left(S_{n}\right)$ for all $t \in\left[0, T_{M}\right]$.

Proof. We know that $S_{1}(t), S_{2}(t)$ are Semigroups of contraction and as $F$ is locally Lipschitz in $w_{n}$ in the space $X$, so we have $\exists T_{M}>0$ and $\left(u_{n}, v_{n}\right)$ is a local solution of $\left(\mathrm{S}_{n}\right)$ on $\left[0, T_{M}\right]$.

Lemma 1. Let $\left(u_{n}, v_{n}\right)$ be the solution of the problem $\left(P_{n}\right)$ such that

$$
u_{n_{0}}(x) \geq 0, v_{n_{0}}(x) \geq 0, \quad x \in \Omega .
$$

Then

$$
u_{n}(t, x) \geq 0 \text { and } v_{n}(t, x) \geq 0, \quad \forall(t, x) \in(0, T) \times \Omega .
$$

Proof. Let $\bar{u}_{n}(t, x)=0$ in $(0, T) \times \Omega \Longrightarrow \frac{\partial \bar{u}_{n}}{\partial t}=0$ and $\Delta \bar{u}_{n}=0$ Then

$$
\frac{\partial u_{n}}{\partial t}-d_{1} \Delta u_{n}-f\left(u_{n}, v_{n}\right)=0 \geq \frac{\partial \bar{u}_{n}}{\partial t}-d_{1} \Delta \bar{u}_{n}-f\left(\bar{u}_{n}, v_{n}\right)
$$

and

$$
u_{n}(0, x)=u_{n_{0}}(x) \geq 0=\bar{u}_{n}(0, x) .
$$

Hence, by the comparison theorem we obtain

$$
u_{n}(t, x) \geq \bar{u}_{n}(t, x)
$$


where from:

$$
u_{n}(t, x) \geq 0 .
$$

by the same method given

$$
v_{n}(t, x) \geq 0 .
$$

then $u_{n}(t, x) \geq 0$ and $v_{n}(t, x) \geq 0$.

\subsection{Global existence of the solution of the system $\left(\mathrm{P}_{n}\right)$}

To prove the global existence of the solution of the system $\left(\mathrm{P}_{n}\right)$ for all nonnegative $t$, it is enough to find an estimate of the solution for everything $t \geq 0$, according to Haraux and Kirane [4], Henry [7] and Routh [18].

For this we give the following lemma according to us shows the existence of an estimate of the solution of $\left(\mathrm{P}_{n}\right)$ in $L^{1}(\Omega)$.

Lemma 2. Let $\left(u_{n}, v_{n}\right)$ the solution of the system $\left(P_{n}\right)$, so it exists $M(t)$ which depends only of $t$, such that for all $0 \leq t \leq T_{M}$, we have:

$$
\left\|u_{n}(t)+v_{n}(t)\right\|_{L^{1}(\Omega)} \leq M(t)
$$

Proof. Of the first and second equation of $\left(\mathrm{P}_{n}\right)$ with:

$$
\frac{\partial}{\partial t}\left(u_{n}+v_{n}\right)-\Delta\left(d_{1} u_{n}+d_{2} v_{n}\right)=f\left(u_{n}, v_{n}\right)+g\left(u_{n}, v_{n}\right)
$$

By taking into account of $\left(\mathrm{H}_{3}\right)$ we have:

$$
\frac{\partial}{\partial t}\left(u_{n}+v_{n}\right)-\Delta\left(d_{1} u_{n}+d_{2} v_{n}\right) \leq C\left(u_{n}+v_{n}+1\right)
$$

Let us integrate on $\Omega$ and apply the formula of Green, we find:

$$
\frac{\partial}{\partial t} \int_{\Omega}\left(u_{n}+v_{n}\right) d x \leq C \int_{\Omega}\left(u_{n}+v_{n}+1\right) d x
$$

so

$$
\frac{\frac{\partial}{\partial t} \int_{\Omega}\left(u_{n}+v_{n}\right) d x}{\int_{\Omega}\left(u_{n}+v_{n}+1\right) d x} \leq C
$$


integrate on $[0, t]$, we find:

$$
\left.\ln \int_{\Omega}\left(u_{n}+v_{n}+1\right) d x\right|_{0} ^{t} \leq C t
$$

thus

$$
\ln \frac{\int_{\Omega}\left(u_{n}+v_{n}+1\right) d x}{\int_{\Omega}\left(u_{n_{0}}+v_{n_{0}}+1\right) d x} \leq C t
$$

which implies:

$$
\begin{aligned}
& \int_{\Omega}\left(u_{n}+v_{n}+1\right) d x
\end{aligned}
$$

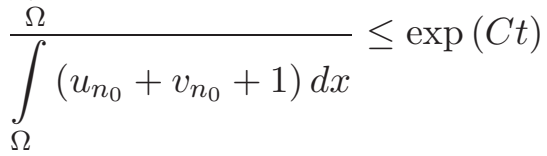

$$
\begin{aligned}
& \Rightarrow \int_{\Omega}\left(u_{n}+v_{n}+1\right) d x \leq \exp (C t) \int_{\Omega}\left(u_{n_{0}}+v_{n_{0}}+1\right) d x \\
& \Rightarrow \int_{\Omega}\left(u_{n}+v_{n}\right) d x \leq \int_{\Omega}\left(u_{n}+v_{n}+1\right) d x \leq \exp (C t) \int_{\Omega}\left(u_{n_{0}}+v_{n_{0}}+1\right) d x \\
& \Rightarrow \int_{\Omega}\left(u_{n}+v_{n}\right) d x \leq \exp (C t) \int_{\Omega}\left(u_{0}+v_{0}+1\right) d x \text { as if } u_{n_{0}} \leq u_{0}, v_{n_{0}} \leq v_{0} \text {. }
\end{aligned}
$$

Let us put:

$$
M(t)=\exp (C t)\left\|u_{0}+v_{0}+1\right\|_{L^{1}(\Omega)}
$$

as $u_{n}, v_{n}$ are positives, then:

$$
\left\|u_{n}+v_{n}\right\|_{L^{1}(\Omega)} \leq M(t), 0 \leq t \leq T_{M}
$$

We can conclude from this estimate that the solution $\left(u_{n}, v_{n}\right)$ given by the theory $\mathbf{3}$ is a global solution. 


\section{Global existence of the solution of the system (1.1)-(1.4)}

We give the following lemma which shows the existence of estimate of the solution $\left(u_{n}, v_{n}\right)$ of a system $\left(\mathrm{P}_{n}\right)$ in $L^{1}(Q)$.

Lemma 3. For any solution $\left(u_{n}, v_{n}\right)$ of $\left(P_{n}\right)$, there is a constant $K(t)$ which depends only of $t$, such that:

$$
\left\|u_{n}(t)+v_{n}(t)\right\|_{L^{1}(Q)} \leq K(t)\left(\left\|u_{0}+v_{0}\right\|_{L^{1}(\Omega)}+1\right)
$$

Proof. To prove this lemma, we use the following results: (see Hollis, Martin and Pierre [8] and Bonafede, Schmitt [2]).

So, we introduce $\theta \in C_{0}^{\infty}(Q), \theta \geq 0$ and $\Phi \in C^{2,1}(Q)$ a nonnegative solution of the following system

$$
\begin{cases}-\frac{\partial \Phi}{\partial t}-d_{1} \Delta \Phi=\theta & \text { on } Q \\ \frac{\partial \Phi}{\partial \eta}=0 & \text { on }[0, T] \times \partial \Omega \\ \Phi(T, .)=0 & \text { on } \Omega,\end{cases}
$$

According to Ladyzenskaya and Solonnikov [13] (S) possesses a unique nonnegative solution. Moreover, for all $q \in] 1,+\infty[$, there exists a nonnegative constant $c$ independent of $\theta$, such that,

$$
\|\Phi\|_{L^{q}(Q)} \leq c\|\theta\|_{L^{q}(Q)}
$$

We have according to Bonafede and Schmitt [2]:

$$
\int_{Q} S_{1}(t) u_{n_{0}}(x)\left(-\frac{\partial \Phi}{\partial t}-d_{1} \Delta \Phi\right) d x d t=\int_{\Omega} u_{n_{0}}(x) \Phi(0, x) d x
$$

and that:

$$
\int_{Q}\left(\int_{0}^{t} S_{1}(t-s) f\left(u_{n}, v_{n}\right) d s\right)\left(-\frac{\partial \Phi}{\partial t}-d_{1} \Delta \Phi\right) d x d t=\int_{Q} f\left(u_{n}, v_{n}\right) \Phi(s, x) d x d s
$$

where from

$$
\int_{Q}\left(S_{1}(t) u_{n_{0}}(x)\right) \theta d x d t=\int_{\Omega} u_{n_{0}}(x) \Phi(0, x) d x
$$

and

$$
\int_{Q}\left(\int_{0}^{t} S_{1}(t-s) f\left(u_{n}, v_{n}\right) d s\right)^{\theta d x d t}=\int_{Q} f\left(u_{n}, v_{n}\right) \Phi(s, x) d x d s
$$


Let us multiply the first equation of (4.1) by $\theta$, and let us integrate on $Q$, by using (5.1) and (5.2), we obtain:

$$
\begin{aligned}
\int_{Q} u_{n} \theta d x d t & =\int_{Q} S_{1}(t) u_{n_{0}}(x) \theta d x d t+\int_{Q}\left(\int_{0}^{t} S_{1}(t-s) f\left(u_{n}, v_{n}\right) d s\right) \theta d x d t \\
& =\int_{\Omega} u_{n_{0}}(x) \Phi(0, x) d x+\int_{Q} f\left(u_{n}, v_{n}\right) \Phi(s, x) d x d s
\end{aligned}
$$

also, we find:

$$
\int_{Q} v_{n} \theta d x d t=\int_{\Omega} v_{n_{0}}(x) \Phi(0, x) d x+\int_{Q} g\left(u_{n}, v_{n}\right) \Phi(s, x) d x d s
$$

therefore:

$$
\begin{aligned}
\int_{Q}\left(u_{n}+v_{n}\right) \theta d x d t & =\int_{\Omega}\left(u_{n_{0}}(x)+v_{n_{0}}(x)\right) \Phi(0, x) d x \\
& +\int_{Q}\left(f\left(u_{n}, v_{n}\right)+g\left(u_{n}, v_{n}\right)\right) \Phi(s, x) d x d s \\
\leq & \int_{\Omega}\left(u_{0}(x)+v_{0}(x)\right) \Phi(0, x) d x \\
& +\int_{Q} C\left(u_{n}+v_{n}+1\right) \Phi(s, x) d x d s
\end{aligned}
$$

Using Holder inequality we deduce

$$
\begin{aligned}
\int_{Q}\left(u_{n}+v_{n}\right) \theta d x d t \leq & \left\|u_{0}+v_{0}\right\|_{L^{1}(\Omega)} \cdot\|\Phi(0, x)\|_{L^{\infty}(Q)} \\
& +C\left\|u_{n}+v_{n}+1\right\|_{L^{1}(Q)} \cdot\|\Phi\|_{L^{\infty}(Q)} \\
\leq & k_{1}\left(\left\|u_{0}+v_{0}\right\|_{L^{1}(\Omega)}+\left\|u_{n}+v_{n}\right\|_{L^{1}(Q)}+1\right) \cdot\|\theta\|_{L^{\infty}(Q)}
\end{aligned}
$$

Since $\theta$ is arbitrary in $C_{0}^{\infty}(Q)$ this implies

$$
\left\|u_{n}+v_{n}\right\|_{L^{1}(Q)} \leq k_{1}\left(\left\|u_{0}+v_{0}\right\|_{L^{1}(\Omega)}+\left\|u_{n}+v_{n}\right\|_{L^{1}(Q)}+1\right)
$$


we take $k=\frac{k_{1}(t)}{1-k_{1}(t)}$ we find:

$$
\left\|u_{n}+v_{n}\right\|_{L^{1}(Q)} \leq k(t)\left(\left\|u_{0}+v_{0}\right\|_{L^{1}(\Omega)}+1\right)
$$

Proof of theorem 1. Let us define the application $L$ by:

$$
L:\left(w_{0}, h\right) \rightarrow S_{d}(t) w_{0}+\int_{0}^{t} S_{d}(t-s) h(s) d s
$$

where $S_{d}(t)$ the semigroup of contraction generated by the operator $d \Delta$, according to the previous result theorem $\mathbf{2}$ and as $S_{d}(t)$ is compact, then the application $L$, is adding two compact applications in $L^{1}(Q)$,

So it was that $L$ is compact $L^{1}(Q) \times L^{1}(Q)$ in $L^{1}(Q)$.

Therefore, there is a subsequence $\left(u_{n_{j}}, v_{n_{j}}\right)$ of $\left(u_{n}, v_{n}\right)$ and $(u, v)$ of $L^{1}(Q) \times$ $L^{1}(Q)$, such that:

$$
\left(u_{n_{j}}, v_{n_{j}}\right) \text { converges towards }(u, v)
$$

Let us now show that $\left(u_{n_{j}}, v_{n_{j}}\right)$ is a solution of $(4.1)$.

We have:

$$
\left\{\begin{array}{l}
u_{n_{j}}(t, x)=S_{1}(t) u_{n_{0}}+\int_{0}^{t} S_{1}(t-s) f\left(u_{n_{j}}(s), v_{n_{j}}(s)\right) d s \\
v_{n_{j}}(t, x)=S_{2}(t) v_{n_{0}}+\int_{0}^{t} S_{2}(t-s) g\left(u_{n_{j}}(s), v_{n_{j}}(s)\right) d s
\end{array}\right.
$$

so it is enough to show that $(u, v)$ verifies $(1.7)$.

it is clear that if $j \rightarrow+\infty$, we have the following limits:

$$
\begin{aligned}
& f\left(u_{n_{j}}, v_{n_{j}}\right) \rightarrow f(u, v) \text { a.e } \\
& g\left(u_{n_{j}}, v_{n_{j}}\right) \rightarrow g(u, v) \text { a.e }
\end{aligned}
$$

and

$$
\begin{aligned}
& u_{n_{0}} \rightarrow u_{0} \\
& v_{n_{0}} \rightarrow v_{0}
\end{aligned}
$$


Thus to show that $(u, v)$ verifies $(1.7)$, it remains to show that:

$$
\begin{aligned}
& f\left(u_{n_{j}}, v_{n_{j}}\right) \rightarrow f(u, v) \\
& g\left(u_{n_{j}}, v_{n_{j}}\right) \rightarrow g(u, v)
\end{aligned}
$$

in $L^{1}(Q)$ when $j \rightarrow+\infty$.

We integrate the first and second equations of $\left(\mathrm{P}_{n}\right)$ on $Q$ by taking into account that:

$$
\begin{aligned}
-d_{1} \int_{Q} \Delta u_{n_{j}} d x d t & =0 \\
-d_{2} \int_{Q} \Delta v_{n_{j}} d x d t & =0
\end{aligned}
$$

we have:

$$
\begin{aligned}
\int_{\Omega} u_{n_{j}} d x-\int_{\Omega} u_{n_{0}} d x & =\int_{Q} f\left(u_{n_{j}}, v_{n_{j}}\right) d x d t \\
\int_{\Omega} v_{n_{j}} d x-\int_{\Omega} v_{n_{0}} d x & =\int_{Q} g\left(u_{n_{j}}, v_{n_{j}}\right) d x d t
\end{aligned}
$$

where from:

$$
\begin{aligned}
& -\int_{Q} f\left(u_{n_{j}}, v_{n_{j}}\right) d x d t \leq \int_{\Omega} u_{0} d x \\
& -\int_{Q} g\left(u_{n_{j}}, v_{n_{j}}\right) d x d t \leq \int_{\Omega} v_{0} d x .
\end{aligned}
$$

Let us put

$$
\begin{aligned}
& N_{n}=C\left(u_{n_{j}}+v_{n_{j}}+1\right)-f\left(u_{n_{j}}, v_{n_{j}}\right) \\
& M_{n}=C\left(u_{n_{j}}+v_{n_{j}}+1\right)-f\left(u_{n_{j}}, v_{n_{j}}\right)-g\left(u_{n_{j}}, v_{n_{j}}\right)=N_{n}-g\left(u_{n_{j}}, v_{n_{j}}\right)
\end{aligned}
$$

it is clear that $N_{n}$ and $M_{n}$ are positives according to $\left(\mathrm{H}_{3}\right)$ and $\left(\mathrm{H}_{4}\right)$, of (5.4) and (5.5) we obtain:

$$
\int_{Q} N_{n} d x d t \leq C \int_{Q}\left(u_{n_{j}}+v_{n_{j}}+1\right) d x d t+\int_{\Omega} u_{0} d x
$$




$$
\int_{Q} M_{n} d x d t \leq C \int_{Q}\left(u_{n_{j}}+v_{n_{j}}+1\right) d x d t+\int_{\Omega}\left(u_{0}+v_{0}\right) d x
$$

the lemma 3 gives us:

$$
\begin{aligned}
& \int_{Q} N_{n} d x d t<+\infty \\
& \int_{Q} M_{n} d x d t<+\infty
\end{aligned}
$$

which implies:

$$
\begin{gathered}
\int_{Q}\left|f\left(u_{n_{j}}, v_{n_{j}}\right)\right| d x d t \leq C \int_{Q}\left(u_{n_{j}}+v_{n_{j}}+1\right) d x d t+\int_{Q} N_{n} d x d t<+\infty \\
\int_{Q}\left|g\left(u_{n_{j}}, v_{n_{j}}\right)\right| d x d t \leq \int_{Q} M_{n} d x d t+\int_{Q} N_{n} d x d t<+\infty
\end{gathered}
$$

let

$$
\begin{aligned}
h_{n} & =N_{n}+C\left(u_{n_{j}}+v_{n_{j}}+1\right) \\
\Psi_{n} & =N_{n}+M_{n}
\end{aligned}
$$

$h_{n}$ and $\Psi_{n}$ are in $L^{1}(Q)$ and positives and furthermore

$$
\begin{aligned}
\left|f\left(u_{n_{j}}, v_{n_{j}}\right)\right| & \leq h_{n} \text { a.e } \\
\left|g\left(u_{n_{j}}, v_{n_{j}}\right)\right| & \leq \Psi_{n} \text { a.e. }
\end{aligned}
$$

Let us combine this result with (5.3) and we apply the theorem of convergence dominated by Lebesgue.

We obtain:

$$
\begin{aligned}
& f\left(u_{n_{j}}, v_{n_{j}}\right) \rightarrow f(u, v) \\
& g\left(u_{n_{j}}, v_{n_{j}}\right) \rightarrow g(u, v)
\end{aligned} \text { in } L^{1}(Q)
$$

by passing in the limit $j \rightarrow+\infty$ of $\left(\mathrm{P}_{j}\right)$ in $L^{1}(Q)$ we find:

$$
\left\{\begin{array}{l}
u(t, x)=S_{1}(t) u_{0}+\int_{0}^{t} S_{1}(t-s) f(u(s), v(s)) d s \\
v(t, x)=S_{2}(t) v_{0}+\int_{0}^{t} S_{2}(t-s) g(u(s), v(s)) d s
\end{array}\right.
$$

Then $(u, v)$ verify (1.7) consequently $(u, v)$ is the solution of (1.1)-(1.4). 


\section{References}

[1] N.D. Alikakos, $\mathrm{L}^{p}$-bounds of solutions of reaction-diffusion equations, Comm. Partial Differential Equations 4 (1979), 827-868.

[2] S. Bonafede, D. Schmitt, Triangular reaction-diffusion systems with integrable initial data, Nonlinear analysis 33 (1998), 785.

[3] T. Diagana, Some remarks on some strongly coupled reaction-diffusion equations, J. Reine. Angew (2003).

[4] A. Haraux, M. Kirane, Estimations $\mathrm{C}^{1}$ pour des problèmes paraboliques semi-lineaires, Ann. Fac. Sci. Toulouse Math. 5 (1983), 265-280.

[5] A. Haraux, A. Youkana, On a result of K. Masuda concerning reactiondiffusion equations, Tohoku Math. J. 40 (1988), 159-163.

[6] D. Henry, Geometric Theory of Semilinear Parabolic Equations. Lecture Notes in Mathematics 840, Springer-Verlag, New-York (1981).

[7] D. Henry, Theory of semilinear parabolic equations, Lecture notes in Math, 840, Springer Verlag, New York (1984).

[8] S.L. Hollis, R. H. Martin And M. Pierre, Global existence and boundedness in reaction-diffusion systems. SIAM J. Math anal, 18: (1987), 744-761.

[9] I. Kanel, M. Kirane, Global existence and large time behavior of positive solutions to a reaction-diffusion system, Differ. Integral Equ. Appl. 13(1-3) (2000), 255-264.

[10] M. Kirane, Global Bounds and Asymptotics for a System of ReactionDiffusion Equations, Journal of Mathematical Analysis and Applications, 138 (1989), 328-342.

[11] S. Kouachi, Global existence of solutions to reaction-diffusion systems via a Lyapunov functional, Electron Jornal of Differential Equations, Vol. 2001 (2001), No. 68, 1-10.

[12] S. Kouachi, A. Youkana. Global existence for a class of reaction-diffusion systems, Bull. Polish. Acad. Sci. Math. 49(3) (2001).

[13] O.A. Ladyzenskaya, V. A Solonnikov, N. N Uralceva, Linear and quasilinear equations of parabolic type, Trans. Math. Monographs. vol. 23, AMS; Providence, R. I (1968). 
[14] R.H. Martin, M. Pierre, Nonlinear reaction-diffusion systems, in: Nonlinear Equations in the Applied Sciences, Math. Sci. Eng. Acad. Press, New York (1991).

[15] K. Masuda, On the global existence and asymptotic behaviour of solution of reaction-diffusion equations. Hokkaido Math, J. 12: (1983), 360-370.

[16] A. Moumeni, L. Salah Derradji, Global existence of solution for reactiondiffusion systems, IAENG, Int. J. Appl. Math. 40(2) (2010), 84-90.

[17] A. Pazy, Semigroups of linear operators and applications to partial differential equations, Springer, New York (1983).

[18] F. Rothe, Global existence of Reaction-Diffusion Systems, Lecture Notes in Math. 1072, Springer, Berlin (1984). 